Résumés des conférences et travaux

\title{
Historiographie de la Renaissance dans le domaine de l'architecture
}

Méthodes et approches, $\mathrm{XIX}^{\mathrm{e}}-\mathrm{XX}^{\mathrm{e}}$ siècles

\section{Antonio Brucculeri}

\section{OpenEdition \\ Journals}

Édition électronique

URL : https://journals.openedition.org/ashp/1018

DOI : 10.4000/ashp.1018

ISSN : 1969-6310

Éditeur

Publications de l'École Pratique des Hautes Études

\section{Édition imprimée}

Date de publication : 2 février 2011

Pagination : 231-232

ISSN : 0766-0677

\section{Référence électronique}

Antonio Brucculeri, «Historiographie de la Renaissance dans le domaine de l'architecture », Annuaire de l'École pratique des hautes études (EPHE), Section des sciences historiques et philologiques [En ligne], 141 | 2011, mis en ligne le 24 février 2011, consulté le 06 juillet 2021. URL : http:// journals.openedition.org/ashp/1018; DOI : https://doi.org/10.4000/ashp.1018 


\title{
HISTORIOGR A PHIE DE LA RENAISSANCE DANS LE DOMAINE DE L'ARCHITECTURE
}

\author{
MÉTHODES ET APPROCHES, XIX ${ }^{\mathrm{e}}-\mathrm{XX}^{\mathrm{e}}$ SIÈCLES
}

\author{
Chargé de conférences : M. Antonio BRUCCULERI
}

Programme de l'année 2008-2009: Historiographie de la Renaissance dans le domaine de l'architecture : méthodes et approches, $X I X^{e}-X X^{e}$ siècles.

Pendant cette première année, le cycle de conférences a fourni aux étudiants un aperçu général sur les enjeux de l'approche historiographique de la Renaissance en architecture à travers l'illustration de quelques cas de figure exemplaires (auteurs et milieux) dans la période 1850-1950. Il a été question de souligner l'intérêt de l'historiographie de la Renaissance dans le domaine de l'architecture en tant que complément essentiel pour comprendre l'évolution des méthodes d'étude et de recherche concernant l'histoire des bâtiments conçus et réalisés dans cette période. La lecture de la genèse de l'histoire architecturale en tant que champ d'études déterminant dans la définition du domaine disciplinaire de l'histoire de l'art et le constat du rapport devenu par la suite dialectique entre deux savoirs - l'histoire de l'art et l'histoire de l'architecture - profondément liés à l'origine, ont constitué les préalables du cycle de conférences.

Loin de toute présentation d'un enchaînement de phases héroïques, ce cycle de conférences a interrogé les thèmes et les contextes à travers lesquels l'historiographie de la Renaissance dans le domaine de l'architecture s'est construite tout au long d'un siècle. L'analyse comparée de différents contextes nationaux (Allemagne, France, Grande-Bretagne et Italie) a été un aspect incontournable de notre démarche. La comparaison a visé le rôle de quelques acteurs / auteurs de la définition d'une approche spécifique de l'histoire de l'architecture des Temps modernes et, plus spécialement, des $\mathrm{XV}^{\mathrm{e}}$ et $\mathrm{XVI}^{\mathrm{e}}$ siècles. À travers les parcours individuels, nous avons par ailleurs souligné les spécificités des itinéraires intellectuels et professionnels en tant que reflet de contextes culturels, sociaux et politiques qui ont marqué les interprétations historiographiques.

La lecture de ces parcours a montré aux étudiants à quel point l'illustration des origines et de l'évolution des méthodes de la discipline engage une large enquête historique. Dans cette perspective, nos conférences ont pris en compte le lien entre le développement de la recherche érudite et la valorisation patrimoniale des bâtiments, l'évolution des rapports entre étude historique des formes architecturales de la Renaissance et critique de l'architecture des $\mathrm{XIX}^{\mathrm{e}}$ et $\mathrm{XX}^{\mathrm{e}}$ siècles, les approches multiples et le caractère mouvant de la périodisation, souvent liés à la mise en évidence d'une période-clé dans l'histoire architecturale d'un pays. En fait, nous avons éclairé les 
différentes manières d'interpréter l'histoire de l'architecture de la Renaissance à ses débuts : nous avons abordé son inscription dans le tableau général de l'histoire architecturale d'un pays, mais aussi sa lecture dans le cadre d'une plus large valorisation des transferts artistiques et culturels à travers le temps et l'espace.

Nous avons d'abord illustré la manière dont le regard initialement adressé à l'architecture de la Renaissance se définit par rapport à la place accordée à l'architecture du Moyen Âge dans l'approche théorique et critique de l'architecture au milieu du XIX ${ }^{\mathrm{e}}$ siècle : dans cette perspective, nous avons abordé et confronté les positions d'Eugène Viollet-le-Duc, de César Daly, de John Ruskin et de Gottfried Semper. Nous avons parallèlement souligné l'attention portée aux édifices et le rôle du relevé dans l'appropriation des monuments de la Renaissance par les architectes : dans cette visée, le cas exemplaire de l'action et de l'œuvre de Paul Marie Letarouilly a été soumis aux étudiants.

Dans un deuxième temps, nous avons évalué l'émergence de l'intérêt des historiens pour l'architecture de la Renaissance à travers les travaux des fondateurs de la discipline de l'histoire de l'art dans le contexte germanophone : des conférences sur l'œuvre de Jacob Burckhardt, de Heinrich Wölfflin et d'Aby Warburg se sont ainsi enchaînées. L'évocation de l'essor de l'histoire de l'art en France entre le dernier quart du XIX ${ }^{\mathrm{e}}$ et le début du XX ${ }^{\mathrm{e}}$ siècle nous a par ailleurs permis de mettre en évidence la construction d'une histoire de l'architecture de la Renaissance, subordonnée à la valorisation des identités nationales.

Pour finir, nous avons consacré un dernier groupe de conférences à trois cas significatifs - en France, en Italie et en Grande-Bretagne - de l'affirmation, pendant la première moitié du $\mathrm{XX}^{\mathrm{e}}$ siècle, d'une historiographie de la Renaissance consacrée à l'architecture. Ces conférences ont porté sur Louis Hautecoeur et son projet historique d'une histoire de 1' « architecture classique » en France ; sur Gustavo Giovannoni et la définition d'une autonomie disciplinaire de l'histoire de l'architecture à l'intérieur de laquelle l'étude de l'architecture de la Renaissance italienne occupe une place de choix ; enfin, sur Rudolf Wittkower, le milieu du Warburg Institute et la genèse - dans le contexte britannique - d'une approche spécifique de l'histoire de l'architecture de la Renaissance entre la seconde moitié des années 1930 et la première moitié des années 1940.

Les étudiants qui ont assisté assidûment aux conférences, parmi lesquels figuraient des étudiants en échange international, ont pris part de manière active aux discussions engagées à la conclusion des interventions. Lors de ces discussions, les questionnements sur les origines de la discipline ont souvent accompagné la réflexion sur les méthodes actuelles. 